\title{
Advances in the development of a Mach-Zehnder interferometric Doppler imager for seismology of giant planets
}

\author{
Ivan Gonçalves ${ }^{1}$, François-Xavier Schmider ${ }^{1}$, Yves Bresson ${ }^{1}$, Julien Dejonghe ${ }^{1}$, Olivier Preis ${ }^{1}$, \\ Sylvie Robbe-Dubois ${ }^{1}$, Thierry Appourchaux ${ }^{2}$, Patrick Boumier ${ }^{2}$, Jean-Christophe Leclec' ${ }^{2}$, Gilles \\ Morinaud $^{2}$, Patrick Gaulme $e^{3,4}$ and Jason Jackiewicz ${ }^{3}$ \\ ${ }^{1}$ Laboratoire Lagrange, Université Côte d’Azur, OCA, CNRS, Nice, France ; \\ ${ }^{2}$ Institut d'Astrophysique Spatiale, Université Paris Sud, CNRS, Orsay, France ; \\ ${ }^{3}$ Department of Astronomy, New Mexico State University, Las Cruces, NM, USA; \\ ${ }^{4}$ Apache Point Observatory, Sunspot, NM, USA
}

\begin{abstract}
The measurements of radial velocity fields on planets with a Doppler Spectro-Imager allow the study of atmospheric dynamics of giant planets and the detection of their acoustic oscillations. The frequencies of these oscillations lead to the determination of the internal structure by asteroseismology. A new imaging tachometer, based on a Mach-Zehnder interferometer, has been developed to monitor the Doppler shift of solar lines reflected at the surface of the planets. We present the principle of this instrument. A prototype was designed and built, following the specifications of a future space mission. The performance of the prototype, both at the laboratory and on the sky, is presented here.
\end{abstract}

Keywords: Giant planets, asteroseismology, radial velocity map, Mach-Zehnder imaging interferometer, Jupiter, acoustic oscillations

\section{INTRODUCTION}

The Doppler Spectro-Imager (DSI) presented in this paper is dedicated to the study of the internal structures and atmospheric dynamics of giant planets. It produces simultaneously radial velocity maps and images in the visible domain of the whole planet surface. Studies of that instrument were initially undertaken for the Laplace space mission, which was selected as an ESA Large mission under the name JUICE (JUpiter ICy moons Explorer). The DSI instrument was proposed to study the formation and evolution of Jupiter's system ${ }^{1}$. Although the instrument was not selected for the final payload of the space mission, the R\&T was continued and a prototype of the instrument was realized in view of ground based observations.

Seismology was the first objective of the instrument, while the study of atmospheric dynamics from radial velocities, independently of cloud-tracking measurement, was an additional target. The design of the instrument has been mainly driven by constraints arising from seismology. Seismology consists in retrieving the internal density profile of a planet thanks to the measurements of its acoustic mode frequencies ${ }^{2}$. Helioseismology has been very successful for the past 40 years to determine the solar internal structure ${ }^{3}$. The possibility to use seismic technique for Jupiter's internal structure has been envisioned at the very beginning of helioseismology by Vorontsov ${ }^{4}$. Further works have shown how much could be learnt about its interior, in particular the unambiguous determination of the core mass and size ${ }^{5}$. Measurements on the latter would give strong insights on giant planets formation and the Solar System in general, since Jupiter has played a crucial role in shaping the solar system with a large mass and rapid formation ${ }^{6}$. Two scenarios are competing to explain its formation: the nucleated instability model assumes accretion of gas around a rocky core ${ }^{7}$, while the gravitational instability model proposes that giant planets formed by gravitational collapse from the protoplanetary disk ${ }^{8}$.

Theoretical works ${ }^{5,9}$ predict that Jovian global oscillations should have a frequency range of [800, 3500] $\mu \mathrm{Hz}$ with 10 to $100 \mathrm{~cm} / \mathrm{s}$ amplitude, values that are comparable to the Sun's values. Several attempts to observe Jovian global modes have been carried out since the mid-1980 $\mathrm{s}^{10,11}$. The major difficulty of seismic observations of Jupiter is related to its rapid rotation $(12.5 \mathrm{~km} / \mathrm{s}$ at the equator), which diminishes the instrument's velocity sensitivity and makes it extremely sensitive to pointing errors. It conducted to develop a specific instrument combining a good spatial resolution and a high 
spectral sensitivity to measure very small Doppler shifts ${ }^{12}$ and to a first positive detection in $2011^{13}$. This first experience permitted to start the DSI instrument study.

This paper describes the DSI instrumental concept and the first experimental results obtained on a prototype of the Mach-Zehnder interferometer which was realized at the Observatoire de la Côte d'Azur (OCA). We first describe the measurement principle, i.e. how we extract a radial velocity field from the interference pattern, and the choice of the optimized working spectral domain. First tests of the prototype were realized on an optical bench at the Observatoire de la Côte d'Azur. The experimental tests demonstrate that most of the theoretical performance is met. Observations were then carried out with a telescope at Calern Observatory, near Nice (France). We present here some results obtained by observing the diffused solar light during the day, and show the bias due to the finite PSF size for observations of Jupiter. We conclude that the concept is now validated and operational for future observation program within the JOVIAL project $^{14}$ and indicates possible improvement of the instrument.

\section{INSTRUMENT PRINCIPLE}

\subsection{Instrumental concept}

The instrument is a compact Mach-Zehnder interferometer with a fixed Optical Path Difference (OPD). It provides images of extended objects as well as velocity maps. The principle is the following: a narrow band of the solar spectrum reflected on the observed object is injected in the MZ. Any Doppler shift of the spectrum will produce a corresponding phase shift of the fringes at the output of the interferometer following the equation:

$$
\varphi=\frac{2 \pi \Delta}{\lambda} \frac{v}{c}
$$

where $\Delta$ is the OPD and $\lambda$ the wavelength.

The instrument produces simultaneously four images with fringes in phase quadrature. The value of the phase (hence, of the velocity) can be determined on each point of the image through the so-called ABCD method ${ }^{15}$. The OPD and the spectral band are chosen to maximize the Signal to Noise Ratio (SNR) of the velocity measurements. A piezo-electric plate allows a modulation of the OPD for calibration.
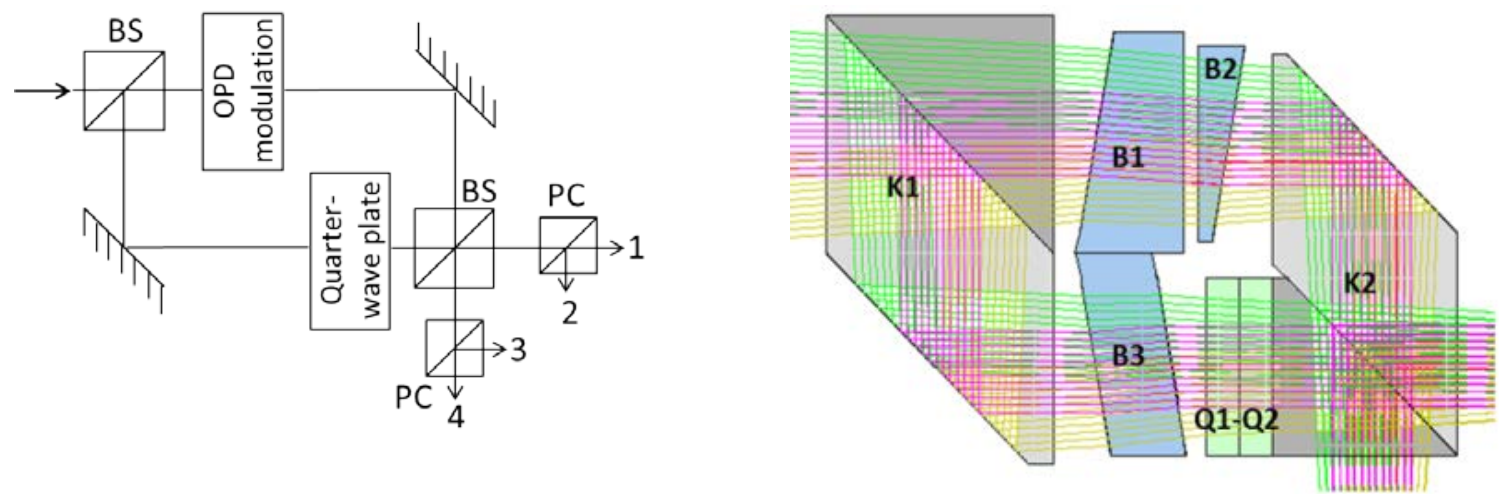

Fig.1. Left: Concept of the Mach-Zehnder interferometer. The first beam splitter produces two identical arms. The second one recombines the beams and provides two outputs with opposite fringe pattern (as long as the beam splitters have no absorption). Each output is separated in two by a Polarizer Cube (PC), providing four beams. Because of the quarter wave plate, the fringe phases of the four outputs are separated by $\pi / 2$. The Optical Path Difference (OPD) can be modulated for calibration. Right: Design of the MZ interferometer. The K1 and K2 blocks, made of K5, constitute an "empty" Mach-Zehnder with a $2 \mathrm{~mm}$ OPD. The tilted plates B1, B2 and B3, made of BK7, produce uniform fringes. B2 is attached to a translation plate that can be moved vertically to change the OPD for calibration purpose. The Q1 and Q2 quartz plates, with a small width difference $(14 \mu \mathrm{m})$ and axes tilted by 90 degrees form a quarter-wave plate that induces a phase shift of $\pi / 2$ between the polarizations. 


\subsection{Measurement principle}

The goal of the instrument is to obtain Doppler shift measurement in each point of the observed object, i.e. it produces radial velocity images. It has been demonstrated that velocity measurements are more sensitive and less noisy than photometric measurements for detection of acoustic oscillations. However, because of the fast rotation of the planets, observations of oscillations on Jupiter or other giant planets requires a good spatial resolution to avoid line broadening that limits the sensitivity and spurious velocity noise coming from inaccurate guiding. The Doppler Specro Imager has that capability, as it produces simultaneously a photometric image of the planet together with a phase map of the fringes at each point of the planet proportional to the Doppler shift of the solar lines.

The standard deviation on the velocity measurement due to photon noise can be expressed as a function of the OPD $\Delta$, the mean wavelength $\lambda$, the contrast $\gamma$ and the number $\mathrm{N}$ of photons per pixel by: .

$$
\sigma_{v}=\frac{c \lambda}{\gamma \pi \Delta \sqrt{2 N}}
$$

It can be seen than the Signal to Noise Ratio can be optimized by maximizing the product of the contrast by the OPD, for a given spectral bandwidth. A systematic exploration of the solar spectrum has been conducted to find the better spectral band. A bandwidth of $1 \mathrm{~nm}$ around $519.4 \mathrm{~nm}$ together with an OPD of $5073 \mu \mathrm{m}$ provides an optimum of the sensitivity, as shown on fig. 2. The Mach-Zehnder has been designed with this OPD.

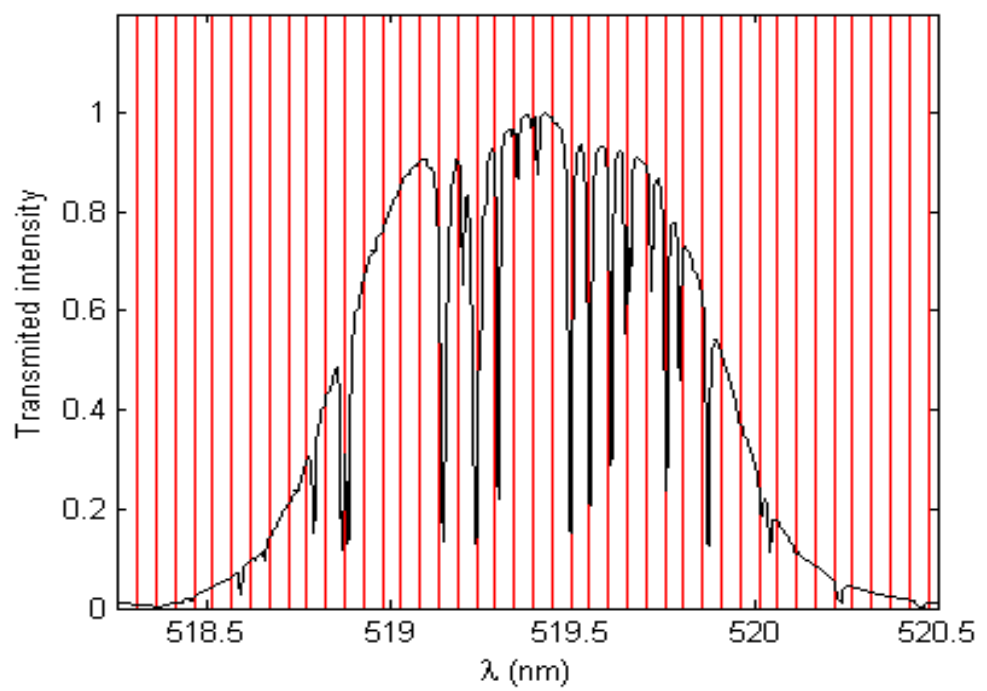

Fig.2. Solar spectrum selected by the entrance filter. The red lines indicate the periodicity of the fringes as a function of the wavelength for the adequate OPD. When they coincide with the spectral lines, the contrast is maximized.

The choice of the OPD and bandwidth conduces to a theoretical maximum contrast of 5\%. However, because of the defaults in the realization of the Mach-Zehnder, the maximum recorded contrast was only $4 \%$ at the center of the field (fig. 4).

\subsection{Data processing}

The phase of the fringes is obtained by the so-called ABCD method: each point of the image of the planet modulated by the fringes is seen at four phases in quadrature:

$$
I_{k}=I_{0}[1+\gamma \cos (\varphi+(k-1) \pi / 2)]
$$


By combining the four images, it is possible to eliminate the flux of the planet and to get the phase as the argument of the complex fringes:

$$
\begin{aligned}
& U=\frac{I_{1}-I_{3}}{I_{1}+I_{3}}=2 \gamma \cos \varphi \\
& V=\frac{I_{2}-I_{4}}{I_{2}+I_{4}}=2 \gamma \sin \varphi
\end{aligned}
$$

and

$$
\varphi=\arg (Z)
$$

$\mathrm{Z}$ being the complex build from $\mathrm{U}$ and $\mathrm{V}$ in the way $\mathrm{Z}=\mathrm{U}+\mathrm{iV}$.

The velocity map can be extracted from the complex fringes $\mathrm{Z}$ by dividing it by $\mathrm{Z}_{\text {instr }}$, where $\mathrm{Z}_{\text {instr }}$ are the instrumental fringes which are measured from a zero velocity field, obtained with a spectral lamp at the laboratory. The full procedure is well described in Soulat ${ }^{16}$.

However, this estimation of the phase as presented above would suppose a uniform pixel response and a perfect quadrature between outputs. In the fact, in order to correctly estimate the phase, we have to correct each sub-images by the pixel response and take into account the departure to quadrature between the outputs. Actually, a model of the fringe pattern for each output is produced from the calibration by modulation of the OPD. The calibration consists in the acquisition of four frames in sequence after increasing the OPD by a value $\lambda / 4$ at each acquisition. This procedure permits to have, for each pixel of the detector, four realizations of the fringes separated by $\pi / 2$.

It is then possible to extract the coefficients of the instrumental fringe pattern for each sub-image in the way described in equation 3 and 4:

$$
I_{k, j}=\tau_{k} I_{0}\left[1+\gamma_{k} \cos \left(\varphi_{k}+(j-1) \pi / 2\right)\right]
$$

and

$$
\begin{gathered}
U_{i n s t, k}=\frac{I_{k, 1}-I_{k, 3}}{I_{k, 1}+I_{k, 3}}=2 \gamma_{k} \cos \varphi_{k} \\
V_{i n s t, k}=\frac{I_{k, 2}-I_{k, 4}}{I_{k, 2}+I_{k, 4}}=2 \gamma_{k} \sin \varphi_{k}
\end{gathered}
$$

The sequence permits to establish for each pixel its photometric response $\tau_{k}$, the local contrast $\gamma_{k}$ and the local phase difference $\varphi_{k}$ to an arbitrary phase reference (assumed to vary linearly within the field).

Some of the calibration maps are displayed on fig. 4.. They show the response of the instrument for the four sub-images of 512x512 pixels on the camera. The detector is a 1024x1024 px E2V CCD. The field diaphragm corresponds to a 60 arcsecs on the sky, allowing the observation of Jupiter. Fig. 4 shows the non-uniformity of the contrast in the field, and the departure to the phase quadrature between sub-images with opposite polarization.

Fig. 5 shows the results of our numerical model of the Mach-Zehnder outputs, taking into account the interferences between the two arms of the Mach-Zehnder on the whole field integrating the solar spectrum on the full bandwidth of the entrance filter. The model includes the possible defaults of the optics and offers the possibility to identify the origin of the different problems found in experimental data. 


\section{EXPERIMENTAL VERIFICATION}
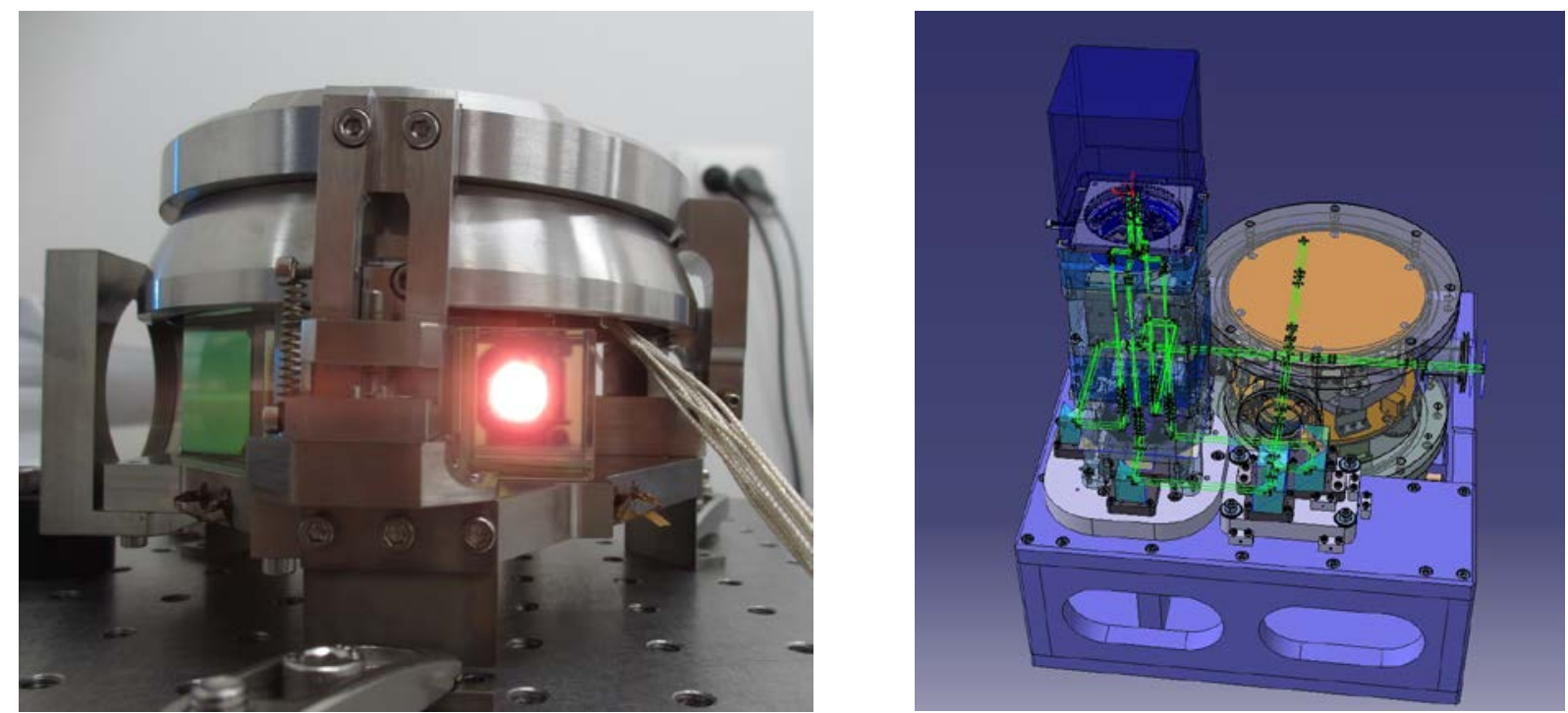

Fig. 3: The Mach-Zehnder interferometer in its mechanical assembly (left). The upper mechanism allows the precise adjustment of the B2 plate inclination on 3 axes. This B2 plate is attached to a piezoelectric driven translation plate, inside this assembly, that permits a precise adjustment of the OPD and its modulation.

On the right, the MZ is placed inside the vaccum tank (in grey) and thermally controlled thanks to a copper oven (in orange) coupled with an external thermal regulator through a circulating fluid. The temperature of the MZ can be set within a range of $30{ }^{\circ} \mathrm{C}$ around room temperature with a stability of $0.1^{\circ} \mathrm{C}$. The two optical outputs of the vacuum tank are separated by polarization beam splitters and folded onto the detector thanks to a series of mirrors.

The Mach-Zehnder interferometer is made of several glass prisms of different materials, all glued on the same Titanium plate, except the moving plate B2, used for adjustment and calibration. The latter is holding on a piezo translation stage, and can be mechanically adjusted in tilt and position with 6 degrees of freedom. The use of the different materials allows a minimization of the thermal sensitivity of the MZ. The MZ is placed in a vacuum tank, thermally controlled to avoid OPD variations due to pressure or temperature variations.

The optical test bench included several light sources: a white lamp, a spectral lamp giving a monochromatic light at $508 \mathrm{~nm}$ and an optical fiber conducting solar light into the laboratory. Several tests were conducted at the laboratory in order to assess the behavior of the instrument. First the wave-front in each arm of the MZ interferometer was analyzed with a Zygo interferometer. This analysis demonstrated that the quality of the wave-front was correct for a beam of 10mm diameter. However, a default of parallelism between the two arms was observed. It appeared after the gluing of the prisms and might have been due to some stress on the glass blocks during the gluing process.

This default is responsible of a loss of contrast inside the Mach-Zehnder, as can be seen on fig. 4. The adjustment process allowed us to partially compensate it by tilting the B2 plate. However, this procedure cannot be perfect and a contrast loss is still present on the size of the field. We were able to model the contrast loss with our numerical model of the $\mathrm{MZ}$, confirm the origin of the default, and providing an optimal set-up of the adjustable B2 plate to have the best possible sensitivity on the whole surface of Jupiter.

We conducted also long-term stability measurement. The MZ was optimized to minimize the sensitivity of the OPD to the temperature. We have checked that the thermal sensitivity shows a minimum for a given temperature, depending on the OPD. However, the piezo-electric plate used for the calibration demonstrated an unexpected sensitivity to the temperature. This sensitivity disappears when the piezo driver is not powered for several days, after an initial drift toward a rest position. This solution is not fully satisfactory as we need to operate the translation plate for calibration purpose at least once every week during the observations. We are presently investigating other piezo translation plates with a different sensor technology and a known temperature response. 

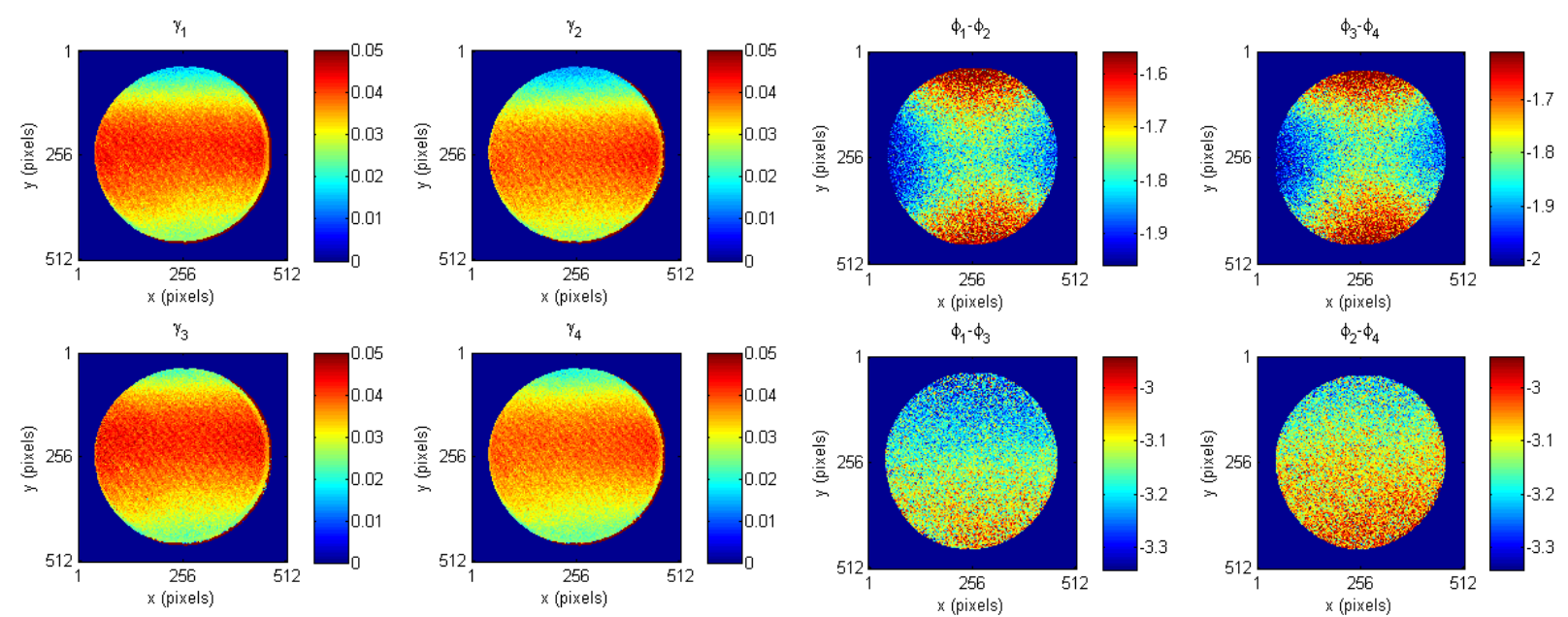

Fig 4. Measured instrumental contrast and phase of the four outputs of the Mach-Zehnder, as obtained by the calibration procedure. Left: the contrast map obtained on solar light for the four fields of the MZ. The two images at the top correspond to the direct output of the MZ, the two at the bottom to the perpendicular output. The two images of the left correspond to one polarization, the two at the right to the perpendicular polarization. The maximum contrast is about $4.3 \%$. The loss of contrast on the edges is due to a misalignment of the optical beams into the MZ (see text). The contrast difference between the different sub-images is mainly due to the non-equilibrium of the beam splitter.

Right: phase differences between outputs of the MZ. The two images on top correspond to phase differences between outputs with opposite polarization (1-2 and 3-4) and the two images below to the phase differences for the outputs with same polarization (1-3 and 2-4). For the top images, the mean phase differences are respectively: 1.76 and $1.81 \mathrm{rad}$, or a discrepancy with the phase quadrature of the order of 10 degree. The shape of the phase response in the field corresponds to the model of the quarter-wave plate (see fig. 5). The two images at bottom have a mean phase difference of $3.15 \mathrm{rad}$, as expected, but the gradient in the image has been identified as the result of a leak of the polarizing beam splitter.
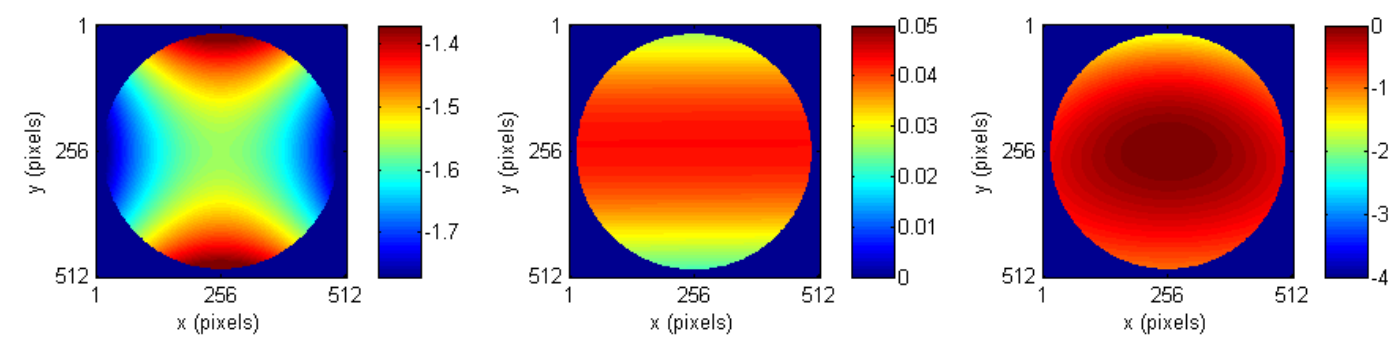

Fig 5. Left : theoretical model of the phase difference between two components of the polarization at the output of the MZ on a field of \pm 2 degrees. The mean value is $\pi / 2$, with departure to the quadrature up to \pm 0.21 radian on the edges. The saddle shape is due to the response of the quarter-wave plate for different input angle. The model reproduces perfectly the observation.

Center : Simulated contrast map obtained with a default of parallelism of the surfaces of the prisms, compensated by an adjustment of tilt of the moving plate B2. The maximum contrast is $4.3 \%$, with a minimum of 2.2 à $2.4 \%$ on the edges. This corresponds to the defaults of the wave-front in the MZ as measured with a Zygo interferometer and reproduces well the contrast measured with the calibration procedure.

Right : Simulated evolution of the phase map along the field, as compared to a regular fringe pattern. The maximum discrepancy is less than $\pi / 2$ radian near the field border.

Nevertheless, we conducted thermal stability tests at the laboratory with the piezo powered in order to assess the phase measurement precision. Despite a higher sensitivity of the MZ to the remaining thermal variations into the vacuum tank, we have shown that the phase measurement variations due to temperature variations were slow enough to not 
compromise the quality of the measurements in the frequency range where the oscillations are expected (fig. 6), as the expected noise level for observations of Jupiter with a $2 \mathrm{~m}$ telescope would be at least $4 \mathrm{~cm}^{2} / \mathrm{s}^{2} / \mu \mathrm{Hz}$.
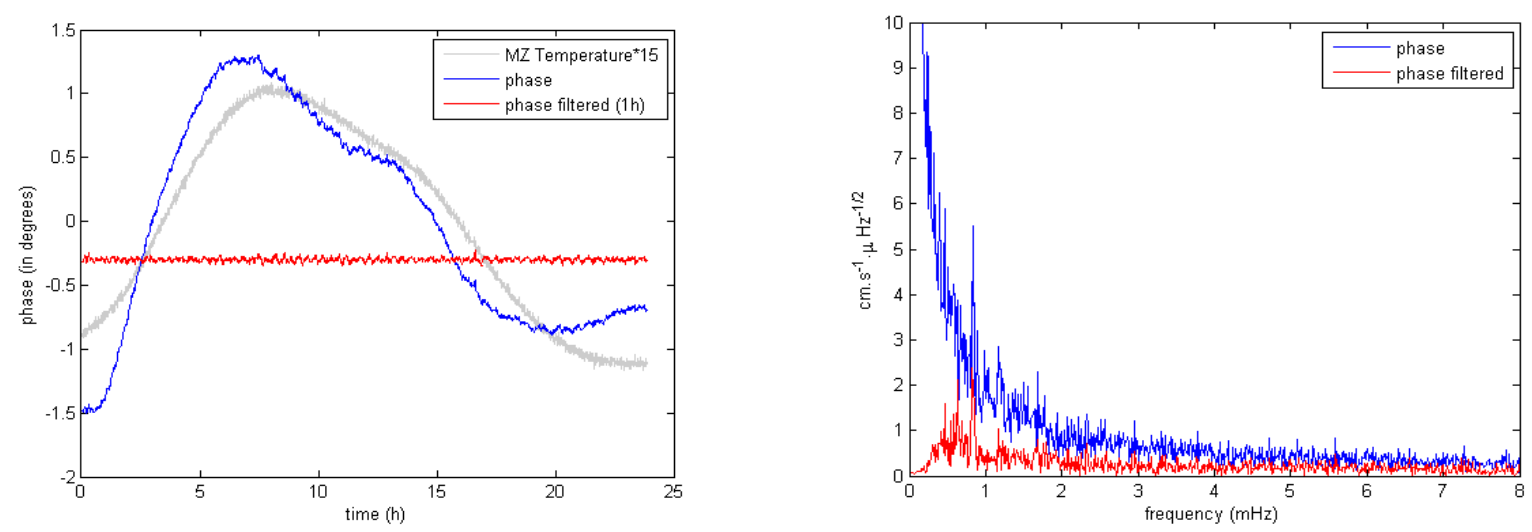

Fig.6. left: The blue curve represents the evolution of the phase with time. After filtering the low frequencies with a one hour high pass filter, the red curve is obtained. The temperature curve (in grey) has been scaled to show the same amplitude variations. The daily temperature variations are about $0,13^{\circ}$ peak to peak. Right : Spectral density of the temporal evolution of the phase converted in velocity with and without low frequency filtering. On the filtered curve in red, the noise level remains lower than the expected photon noise for Jupiter measurements in the acoustic modes frequency range (0.5 to 3 $\mathrm{mHz})$.

\section{TESTS ON THE SKY}

The DSI prototype was installed in the coudé room, at the bottom of the MEO 1.5m telescope at Calern observatory (see above). The optical interface to the telescope included a fast tip-tilt mirror to compensate the guiding default of the telescope and the atmospheric agitation of the image. The red part of the beam (above $550 \mathrm{~nm}$ ) was used to feed a fast guiding camera. The centroid of the planet of the guiding camera was calculated at a rate of $200 \mathrm{~Hz}$ and used as a correction signal to be applied to the steering mirror. This allowed a stabilization of the position of Jupiter in the field within 0.1 arcsec. However, slow drifts of the pupil during the rotation of the telescope occurred. This was responsible of slow variations of the phase, as the instrumental phase of the MZ is not uniform along the field. In a further version of the instrument, a correction of the pupil was implemented by placing a tip-tilt mirror in the first image plane. A stability of the pupil position, better than $1 / 100$ of the pupil diameter, was achieved.
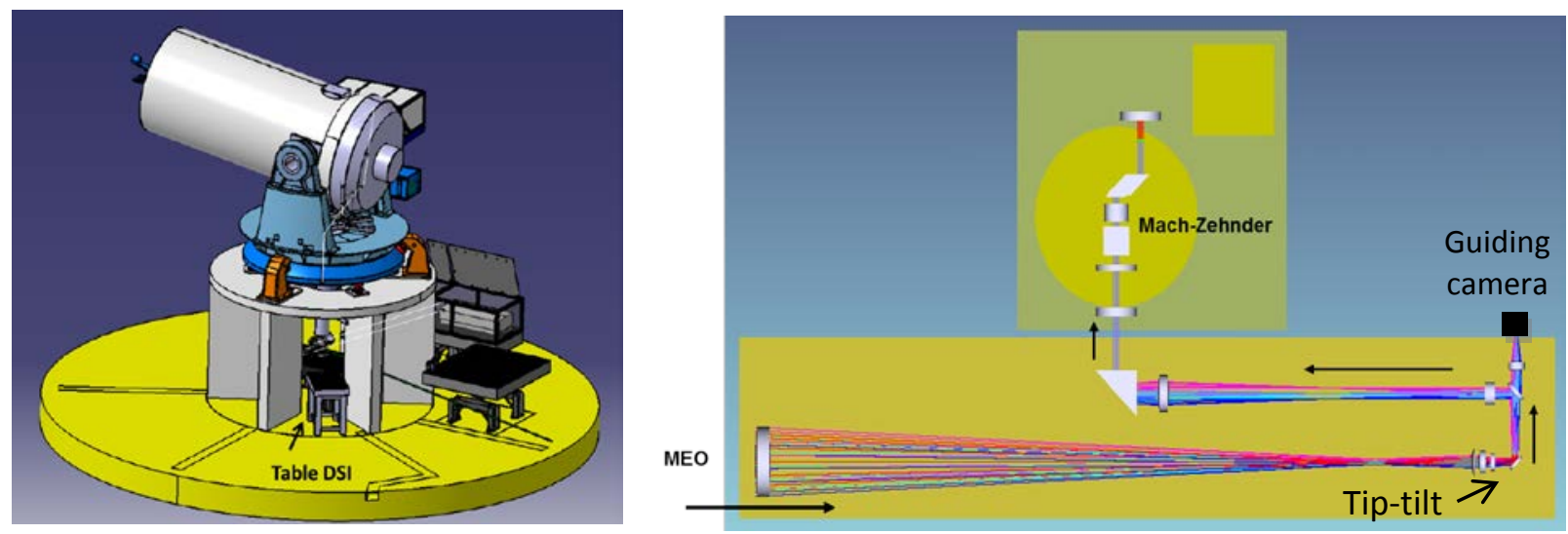

Fig.7. Set-up of the DSI prototype at MEO observatory. The DSI table was placed below the pilar and fed by the an orientable mirror in the azimuthal axis of the telescope. At the right, a schematic view of the optical interface between the telescope after the orientable mirror and the DSI instrument on the board. 
Two types of measurements were made during the tests. First, we observed the diffused daylight through the telescope. The daylight has the same spectral content than the reflected solar light on the planets, but produces a uniform velocity field, with a known velocity between the observer and the Sun. This is very useful to calibrate the instrumental response and to measure the instrument stability. The telescope was then maintained at a fixed position toward the northern horizon. Instrumental calibration produced similar results than the ones obtained at the laboratory.
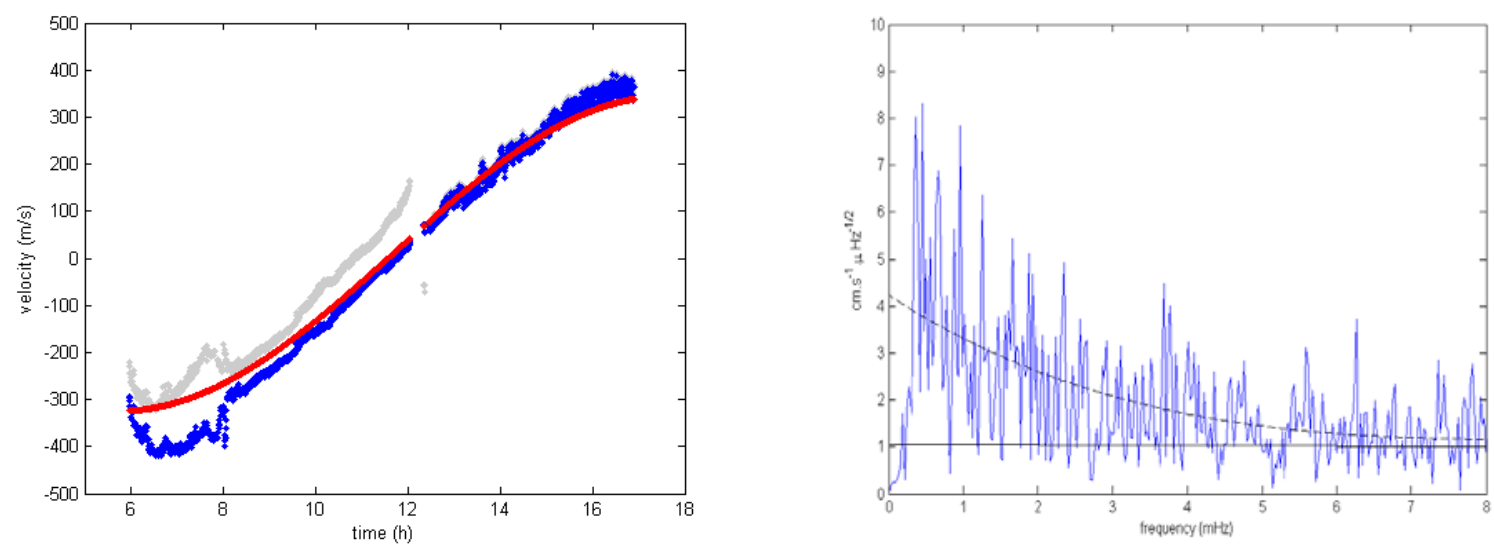

Fig.8. Left: temporal evolution of the phase along the day by observing the diffused skylight. The grey curve represents the raw phase evolution. During the morning, the phase is strongly affected by the variations of the daylight polarization. During the afternoon, only one polarization was selected at the entrance of the instrument. The blue curve is corrected from the bias due to the polarization rate. The Earth-Sun velocity in red varies from $-370 \mathrm{~m} / \mathrm{s}$ to $+370 \mathrm{~m} / \mathrm{s}$ because of Earth rotation. Right: Spectral density of the temporal evolution of the phase converted to velocity, after subtraction of the Earth rotation and low frequency (longer than 1h) filtered out. The horizontal line indicates the level of white noise at high frequency. The dashed line is a quadratic fit of the mean noise level as the inverse of the frequency. The high noise level at low frequencies is due to polarization fast variations with cloud motion in the sky.

The tests put in evidence the effect of the polarization rate at the entrance of the instrument. As the telescope is looking always in the same sky direction, the polarization rate evolves between 0 and $100 \%$ polarization rate following the position of the Sun with respect to the telescope direction on the sky, when looking at blue sky. At first order, the polarization induces a known bias on the phase within the field. However, this bias is dependent of the polarization rate, which can be deduced from the measurements itself, but also of the direction of polarization, which is unknown. Moving white clouds in front of the telescope produce fast variations of the polarization rate and direction that cannot be fully corrected.

Other tests were realized on Jupiter, used as a known velocity field. The fast rotating planet is a good target to test the sensitivity of the instrument and look for possible bias. The velocity field for a single acquisition of 30s on Jupiter clearly shows the fast rotation of the planet, the blue part coming toward the observer. The measured rotation rate is 12.5 $\mathrm{km} / \mathrm{s}$ and is accurate to about $1 \%$ (the error mainly comes from the uncertainty on the size of the planet on the detector). The noise level for each pixel of 0.16 arcsec and for a single exposure of $30 \mathrm{~s}$ is about $1 \mathrm{~km} / \mathrm{s}$ in average. It confirms the theoretical value for the photon noise level. However, a bias is observed on the right and left edge of the image. The bias has been identified and modeled as an effect of the PSF and the non-uniform intensity in the image. In our case, the size of the PSF is dominated by the atmospheric turbulence, and is of the order of 1.5 arcsec (varying from day to day). The correction of the bias is possible if the size and shape of the PSF is well known, which is not always the case. The PSF is presently determined from the data itself by comparison to a high resolution image of Jupiter taken at the same period. An Adapative Optics system not only would provide a much better spatial resolution and lower velocity bias, but also it would permit to known the PSF at any moment for further correction.

\section{CONCLUSION}

We developed a new kind of imaging tachometer, dedicated to the study of giant planets interior and atmosphere dynamics through the measurement of surface velocity maps. In order to detect Jovian oscillations, the specifications for is to have a velocity noise level lower than $4 \mathrm{~cm}^{2} / \mathrm{s}^{2} / \mu \mathrm{Hz}$ in the frequency range of the oscillations, i.e. between 0.8 and 3 
$\mathrm{mHz}$ (3 to $20 \mathrm{mns}$ ). A prototype of the Doppler Seismic Imager has been build and tested at the laboratory, then directly on the sky with a $1.5 \mathrm{~m}$ telescope at Calern observatory. We estimated the performance both at the laboratory and on the sky.

At the laboratory, we were able to check the correctness of the instrumental set-up and calculate the noise level for each image and the phase stability during the time. The calibration procedure permits to establish the instrumental phase map that will be used for processing of the data. The instrumental phase map shows some defects, in particular a non-uniform contrast and a departure to the phase quadrature between polarizations. We were able to model the observed defects, taking into account the misalignments into the MZ, also recorder with a Zygo interferometer. Thanks to this work, we have developed a new procedure for the gluing of the MZ assembly that will avoid this contrast loss. The polarization beam splitters used for the prototype proved to have an insufficient field. New polarizers have been provisioned to circumvent that default. The assessment of the long-term stability of the phase measurement was one of the objectives of the laboratory tests. Instrumental noise as measured at the laboratory is smaller than the expected photon noise on the scientific measurement in the frequency range of acoustic modes.
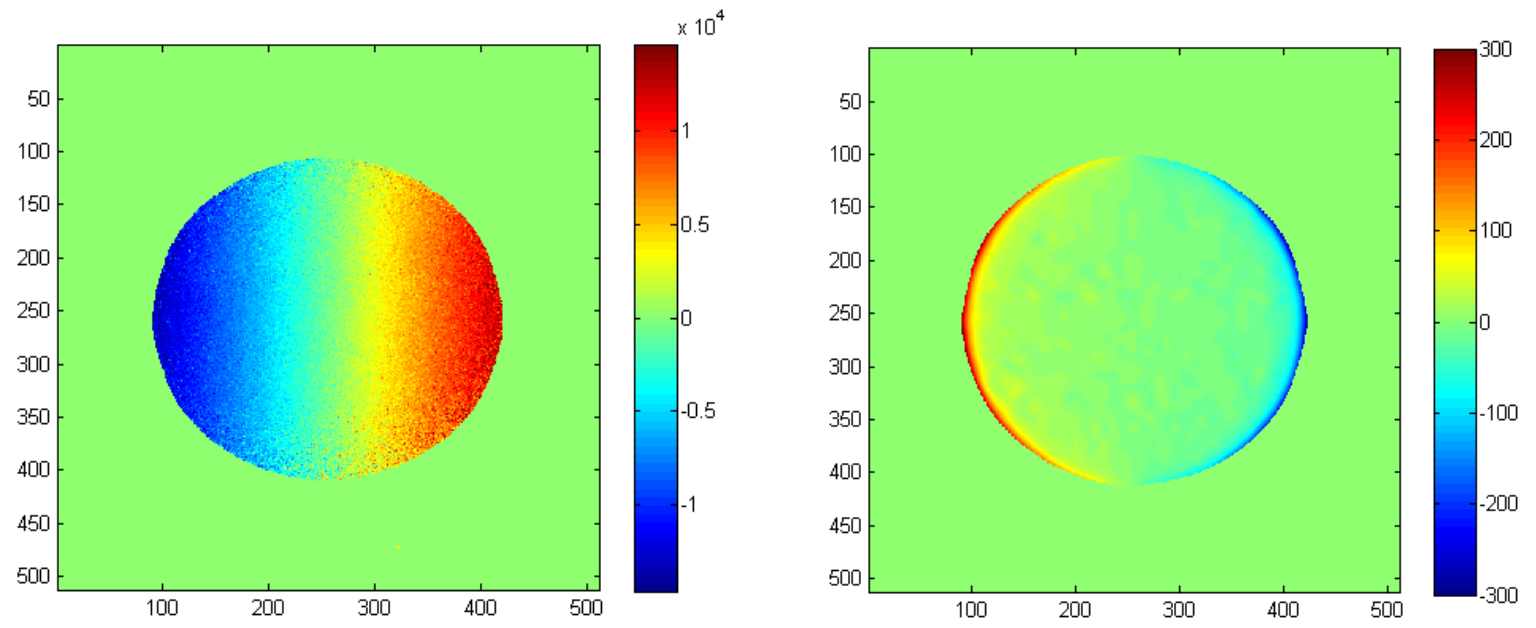

Fig.9. Left: velocity field of the rotation of Jupiter obtained for one single acquisition. Right: bias between recorded and theoretical velocity map of Jupiter due to the finite PSF size (here about 1.5 arcsec) which produces an underestimation of the velocity at the edges of the planet. The color scale is in $\mathrm{m} / \mathrm{s}$ for both figures.

We then installed the instrument at the Coudé focus of the MEO telescope at Calern observatory in order to test the instrument on the sky, both on diffused solar light during the day and on Jupiter during the night. Slow drifts (with periods longer than 1h) occur for observations with the telescope, due to motions of the optical beam and changes in the polarization rate. We minimized the drifts by stabilizing the pupil at the entrance of the instrument and intent to correct the polarization effect. With this precaution, the stability of the instrument during daylight measurement allows to clearly see the Earth rotation motion and to estimate the noise level. This noise level is compatible with the laboratory performance at high frequency. Low frequency noise $(<3 \mathrm{mHz})$ was dominated by cloud motion in the sky which induced fast polarization variations. It was possible to minimize that effect by placing a polarizer at the entrance of the instrument but to the cost of losing $75 \%$ of the photons, the white noise level is then multiplied by a factor of 2 . With this set-up, the solar oscillations could be detectable by looking at daily stray light for about 4 days. It has to be noticed that such effect would not occur with observations of Jupiter, which has very low polarization in the bandwidth of our instrument, except very near the poles.

We foresee to change the polarizer beam splitters and to optimize the light path from the telescope to the instrument to minimize the polarization variations during the night observations.

Observations of Jupiter showed for the first time Doppler images of the fast rotation of the planet. The measured sensitivity to the velocity field is accurate to about $1 \%$ in average. A strong bias in the measured velocity map appeared at the edge of the planet. It is identified as an effect of the PSF, mainly dominated by the atmospheric turbulence. This 
effect could be modeled and corrected. Other improvements are presently being implemented. In particular, a large field Adaptive Optics (AO) system will be used. It will permit a partial correction of the atmospheric turbulence effect. It will improve the resolution of the images and minimize the velocity bias due the size of the PSF.

Two more copies of the instrument will be realized within the JOVIAL project, supported by the French Agence Nationale de la Recherche (ANR) and NASA/EPSCOR funds. Observations of Jupiter with three telescopes around the world (USA, France, Japan) to obtain continuous measurements for several weeks are foreseen in 2018.

\section{REFERENCES}

[1] Soulat, L., Schmider F.-X. et al, "Echoes: a new instrumental concept of Doppler Spectro-Imager for the ESA mission projet JUICE,” Optical Complex Systems: OCS11, Proceedings of the SPIE, vol. 8172, 81720V (2011)

[2] Jackiewicz, J., Nettelmann, N., Marley, M., Fortney, J., "Forward and inverse modeling for jovian seismology," Icarus 220, 84 (2012)

[3] Christensen-Dalsgaard, J., "Physics of solar-like oscillations,” Solar Physics, Volume 220, Issue 2, pp.137-268 (2004)

[4] Vorontsov S., Zharkov V., lubimov V.M., “The free oscillations of Jupiter and Saturn,” Icarus, vol. 27, p.109118 (1976)

[5] Gudkova, T. et al, "Seismological comparison of giant planet interior models,” A\&A, 303,594 (1995)

[6] Cameron, A.G.W., Moon and Planets, 18, 5 (1978)

[7] Safronov V.S., \& Ruskol. E. L., Icarus, 49,284 (1982)

[8] Mayer, L., Quinn T., Wadsley J., \& Stadel J., Science, 298, 1756 (2002)

[9] Bercovici, D.; Schubert, G., "Jovian seismology," Icarus 69, p. 557-565 (1987)

[10] Schmider, F.-X.; Fossat, E.; Mosser, B., "Possible detection of Jovian global oscillations," A\&A 248, 281 (1991)

[11] Mosser, B., Mekarnia D., Maillard J-P. et al, "Seismological observations with a Fourier transform spectrometer - Detection of Jovian oscillations," A\&A 267, 604 (1993)

[12] Schmider, F.-X., Gay, J. et al, "SYMPA, a dedicated instrument for Jovian seismology. I. Principle and performance,” A\&A, 474, 1073 (2007)

[13] Gaulme, P., Schmider, F.-X. et al,” Detection of Jovian seismic waves: a new probe of its interior structure” A\&A, 531, 104 (2011)

[14] Schmider, F. X., Appourchaux, T., Gaulme, P. et al, “The JOVIAL Project for Jovian Seismology,” in Fifty Years of Seismology of the Sun and Stars, ASP Conference Proceedings, Vol. 478, 119 (2013)

[15] Wyant J.C., "Use of an ac heterodyne lateral shear interferometer with realtime wavefront correction systems," Applied Optic, 14, 2622-2626 (1975)

[16] Soulat, L.; Schmider, F.-X.; Robbe-Dubois, S.; Appourchaux, T.; Bresson, Y.; Daban, J.-B.; Gaulme, P.; Gay, J.; Gouvret, C., "Echoes: a new instrumental concept of spectro-imaging for Jovian seismology," Proc. SPIE 8442, 84424M (2012)

\section{AKNOWLEDGEMENT}

The $\mathrm{PhD}$ of I. Gonçalves is funded by a grant of the Observatoire de la Côte d'Azur. The DSI prototype development has been supported by CNES and Thalès Alenia Space. The JOVIAL project is funded by the French Agence National pour la Recherche (ANR). The development of the JIVE instrument in New Mexico is supported by NASA/EPSCOR program and by the New Mexico State University. The observations at Calern observatory have been achieved with the help of the MEO telescope team and of the C2PU telescope team. 